\title{
Analysis of quasi-reversible cyclic voltammograms: Transformation to scan-rate independent form
}

\author{
Tamás Pajkossy \\ Institute of Materials and Environmental Chemistry \\ Research Centre for Natural Sciences, Hungarian Academy of Sciences \\ Magyar tudósok körútja 2, Budapest, Hungary, H-1117 \\ e-mail: pajkossy.tamas@ttk.mta.hu
}

\begin{abstract}
A simple procedure is suggested by which cyclic voltammograms, CVs, pertaining to partially diffusion controlled charge transfer reactions can be analyzed. Using this procedure, from a set of CVs taken at varied scan rates, two scan-rate independent, hysteresis-free functions can be calculated. One of them is the diffusion-free polarization curve, the other is the semiintegrated form of the reversible $\mathrm{CV}$.
\end{abstract}

\section{Introduction}

Cyclic voltammetry (CV) is a widely used experimental method for studying kinetics of electrode processes: the current density, as a function of time, $j(t)$ is measured as a function of time-varying potential, $E(t)$. The latter is scanned between two limits with constant speed, $v=d E / d t$; the CVs are the $j(E)$ curves. Both $j(t)$ and $j(E)$ are complicated functions of $v$; hence comparison of two $\mathrm{CV}$ s measured with different $v$ is far from being trivial.

In rare, simple cases, however, there exist mathematical transformations by which CVs taken with different scan rates can be transformed to the one-and-the-same $T(E)$ function - which function does not "remember" the actual form of $E(t)$, it has hysteresis-free, scan rate independent form. That is, $T(E)$ is a state function. To illustrate this statement, two typical cases are worth to be mentioned: ( $i$ ) In the case when double-layer charging proceeds only, the $d c$ capacitance defined as $j(E) / v$ is independent of $v$; (ii) The CVs of reversible redox couples can be transformed to hysteresis-free polarograpic-wave shaped curves using semiintegration. In these two cases the scanrate normalized and semiintegrated currents, respectively, are the scan-rate independent representations of the measured data.

In contrast to the case of the reversible CVs, the CVs of slower redox systems, called quasireversible $\mathrm{CVs}$ cannot be transformed to a single $T(E)$ function. However, as shown in this paper, by measuring a set of quasi-reversible CVs with different scan rates, two such state functions can be obtained by a simple procedure. One of them characterizes charge transfer kinetics, the other the diffusion. Having derived the relevant equations, the transformation is tested with simulated quasireversible $\mathrm{CV}$ curves.

\section{Theory}

Consider a CV measurement of a quasi-reversible redox system [1] with the condition that only the reduced form is present. This species takes part in an $n$-electron, single-step charge transfer reaction on the electrode. Were the charge transfer not hindered by slow diffusion, the current density would be expressed by the 


$$
j_{\text {inf }}(E)=k(E) n \mathrm{~F} c_{\text {red }}
$$

equation where the subscript inf refers to the infinite transport rate, $c_{\text {red }}$ is the bulk concentration of the reduced species, and the rate coefficient $k(E)$ is an exponential function of potential. Whenever $k(E)$ is sufficiently high, the reaction becomes diffusion controlled, and the surface concentrations of both the reduced and the generated oxidised species ( $c_{\text {red }}^{s}$ and $c_{\mathrm{ox}}^{s}$, resp.) differ from those in the bulk. Time-dependence enters the picture just because the concentration differences between surface and bulk, i.e. because of transport hindrance. Then, the time and potential dependence of the current density, $j(E, t)$ is expressed by the following form of the Butler-Volmer law:

$$
j(E, t)=n \mathrm{~F} k_{0}\left[c_{\text {red }}^{\mathrm{s}}(t) \exp \left(\alpha_{\mathrm{a}} \mathrm{F}\left(E-E_{0}\right) / \mathrm{R} T\right)-c_{\mathrm{ox}}^{\mathrm{s}}(t) \exp \left(-\alpha_{\mathrm{c}} \mathrm{F}\left(E-E_{0}\right) / \mathrm{R} T\right)\right]
$$

Here the other-than-mentioned symbols are of their usual meaning.

The CVs of such systems have been theoretically analyzed by a number of authors decades ago; here the starting point is a result of such a paper, Ref. [2]. The 4th equation on page 181 therein expresses how the the rate coefficient of the charge transfer, $k(E)$, should be calculated from $j(E)$. With little changes (oxidation rather than reduction is considered, and some of the symbols has been changed to adhere to the usage of recent related communications $[3,4,5]$ ) that equation reads as

$$
k(E)=D_{\text {red }}^{1 / 2} \cdot j(E) /\left[M_{1}-M(E) \cdot\left(1+\exp \left(-n \mathrm{~F}\left(E-E_{1 / 2}\right) / \mathrm{R} T\right)\right)\right]
$$

Here $E_{1 / 2}$ and $D_{\text {red }}$ are the half-wave potential of the redox pair (see Ref. 1, Ch.5) and the diffusion coefficient of the reduced species, respectively. $M(E)$ and $M_{1}$ needs explanation: $M(E)$ is the socalled semiintegral of the $j(t)$ current density at the actual $E(t)$ potential; the $M(t)$ semiintegral is calculated by the following convolution:

$$
M(t)=\frac{1}{\sqrt{\pi}} \int_{-\infty}^{t} \frac{j(\tau) d \tau}{\sqrt{t-\tau}}
$$

The $M(t)$ function has special relevance in the case of CVs of reversible systems, that is, when the charge transfer in both directions is infinitely fast. Whereas the reversible CVs exhibit a characteristic hysteresis, $M(t)$ plotted against $E(t)$ is a scan-rate independent, hysteresis-free curve, $M_{\text {rev }}(E)$. The properties of $M_{\text {rev }}(E)$ has first been analyzed by Oldham [6]; for a detailed analysis see his recent textbook [4]. From the present point of view important is the

$$
M_{\text {rev }}(E)=M_{1} /\left[1+\exp \left(-n \mathrm{~F}\left(E-E_{1 / 2}\right) / \mathrm{R} T\right)\right]
$$

equation where

$$
M_{1}=n \mathrm{~F} c_{\text {red }} \sqrt{D_{\text {red }}}
$$

that is, $M_{1}$ is the limiting value of $M_{\text {rev }}$ for $E \gg E_{1 / 2}$. Note that alternative forms of Eq.5 also exist:

$$
M_{\text {rev }}=M_{1} / 2 \cdot\left[1+\tanh \left(n \mathrm{~F}\left(E-E_{1 / 2}\right) / \mathrm{R} T\right)\right] \text { and } E=E_{1 / 2}+(\mathrm{R} T / n \mathrm{~F}) \cdot \ln \left[M_{\text {rev }} /\left(M_{1}-M_{\text {rev }}\right)\right] \text {. }
$$

Since just as $j_{\text {inf }}(E), M_{\text {rev }}(E)$ does not depend on time-dependent variables, these two are scan-rate independent forms of charge transfer and diffusion, respectively.

Combining Eqs. 1, 3, 5 and 6, we get the following equation:

$$
j(E(t))=j_{\text {inf }}(E)-\frac{j_{\text {inf }}(E)}{M_{\text {rev }}(E)} \cdot M(E(t))
$$

Note that here only $j$ and $M$ are time-dependent quantities. 
Assume a set of CVs measured at different scan rates $v$; consider points at one and the same $E=\varepsilon$ potential. At that potential both $j$ and $M$ will be different for the different scan rates, so Eq.7 can be written as

$$
j(\varepsilon, v)=j_{\text {inf }}(\varepsilon)-\frac{j_{\text {inf }}(\varepsilon)}{M_{\text {rev }}(\varepsilon)} \cdot M(\varepsilon, v)
$$

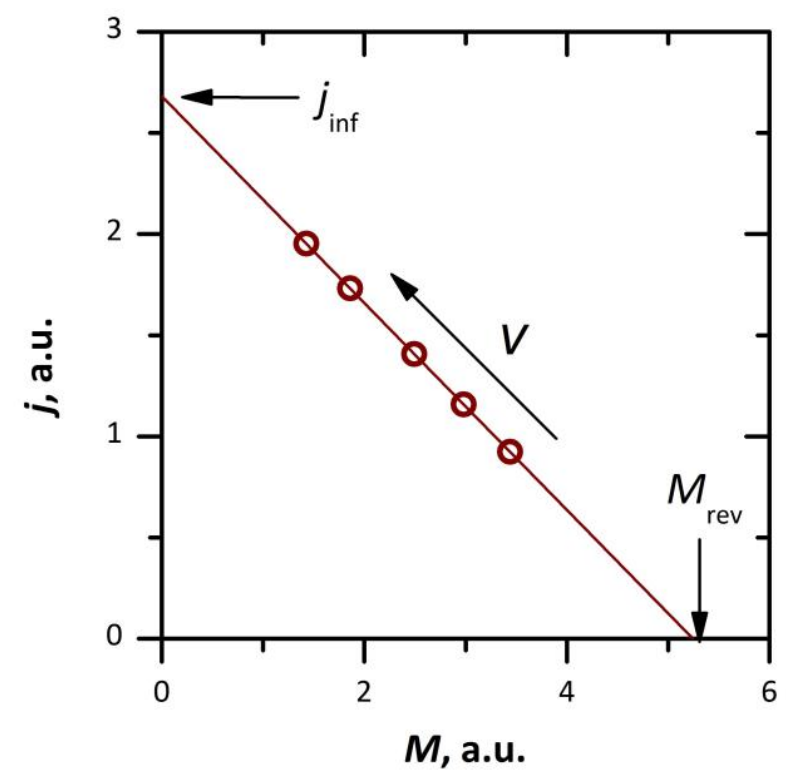

Fig.1. Properties of the $j(\varepsilon)$ vs $M(\varepsilon)$ plot (cf. Eq. 8)

That is, for any $\varepsilon$, a linear relationship $\left(j(v)=\right.$ const $_{1}+$ const $\left._{2} \cdot M(v)\right)$ exists between $j$ and $M$. Hence, if we measure CVs at different scan rates, calculate the semiintegrals, plot the $j-M$ point pairs of the same $\varepsilon$ for all the scan rates, then a straight line is expected. Using e.g., a linear least mean squares $(l m s)$ procedure the intercept and slope can be obtained, from which $j_{\text {inf }}(\varepsilon)$ and $M_{\text {rev }}(\varepsilon)$ values can be calculated. This is graphically expressed in Fig.1. Repeating this procedure for all $\varepsilon \mathrm{s}$, we get $j_{\text {inf }}(E)$ and $M_{\text {rev }}(E)$ functions.

\section{Results of numerical tests}

For testing the properties of the transformation of Eq.8, simulated CVs were generated by a computer program. This was based on the explicit Euler method for solving the partial differential equation relevant to the transport of the redox species [7], taking also into account the quasireversible charge transfer [8] and solution resistance [9].

A set of simulated first-cycle CVs, for five scan rates is displayed in Fig.2a. First, semiintegrated forms were calculated using the algorithm of Ref. 10; these are shown in Fig.2b. Second, the datasets were re-organized to have $j-M$ data pairs at the same potential. Third, according to Eq.8, straight lines were fitted to these points by $l m s$. Finally from the fitted slopes and intercepts $M_{\text {rev }}$ and $j_{\text {inf }}$ values vere calculated for each potential. The obtained $j_{\text {inf }}(E)$ and $M_{r e v}(E)$ functions are shown in Fig.2c. Both curves are hysteresis-free; the characteristic values of the curves: $M_{1}, j$ and the $d \log (j) / d E$ slope at $E=0$ are exactly the same as can be calculated from the input data. Whereas hysteresis or scatter of the points on the $M_{\text {rev }}(E)$ curve is invisibly small, the $\log \left(j_{\text {inf }}(E)\right)$ 
exhibits scatters and deviations from the straight line close to the vertex potentials. In these ranges $j_{\text {inf }}(E)$ is either too large or too small, causing numerical errors in the calculations.
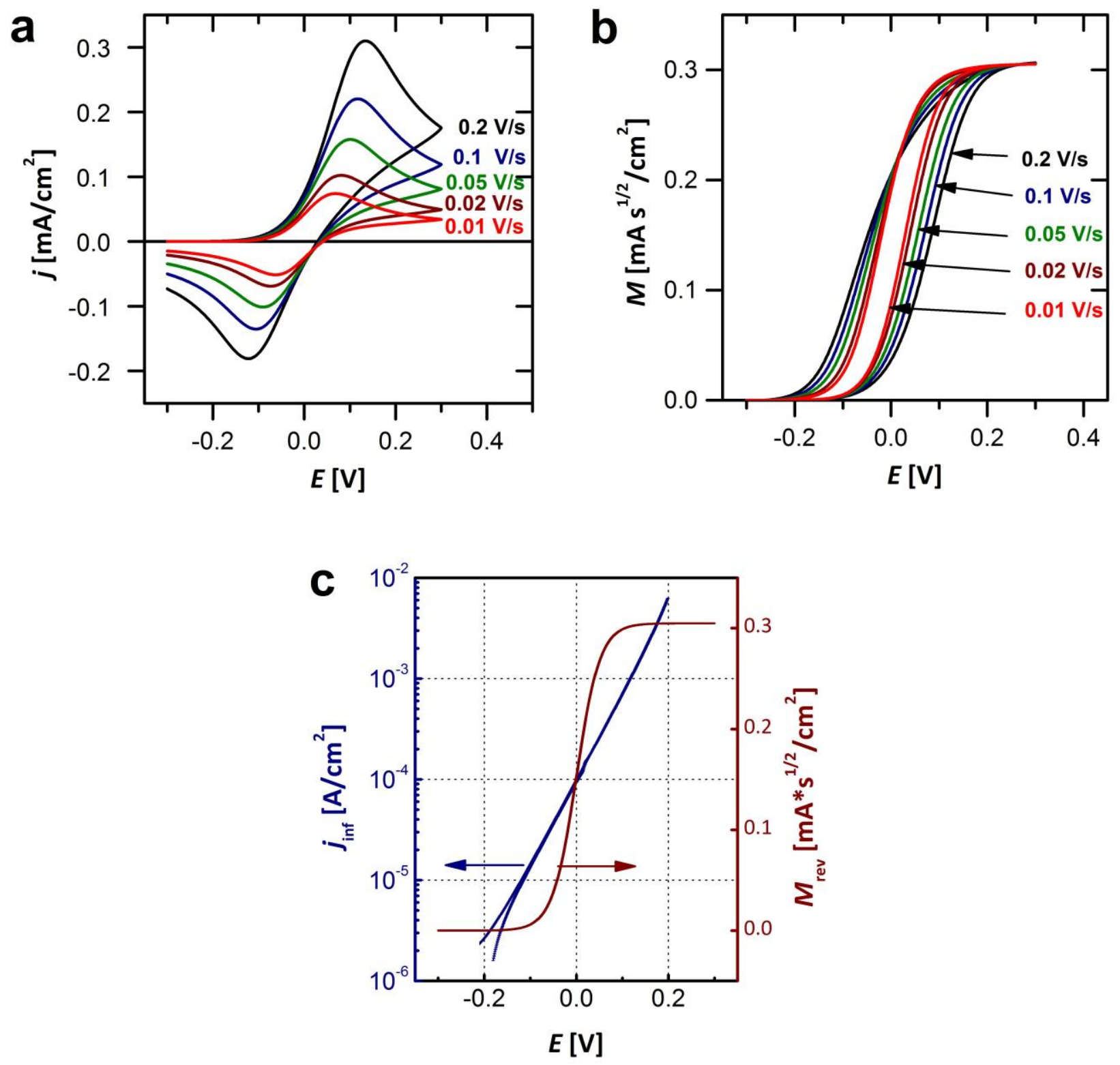

Fig.2. (a) Simulated CVs. Simulation parameters: $D_{\text {red }}=D_{\text {ox }}=10^{-5} \mathrm{~cm}^{2} / \mathrm{s}, \quad c_{\text {red }}=10^{-6} \mathrm{~mol} / \mathrm{cm}^{3}$, $c_{\text {ox }}=0 \mathrm{~mol} / \mathrm{cm}^{3}, E_{0}=0 \mathrm{~V}, k_{0}=0.001 \mathrm{~cm} / \mathrm{s}, n=1, \alpha_{\mathrm{a}}=\alpha_{\mathrm{c}}=0.5, v$ as indicated. (b). The semiintegrated CVs. (c) The $j_{\text {inf }}(E)$ and $M_{r e v}(E)$ functions. 


\section{Discussion}

1. Apparently Eq. 8 is a simple combination of four equations known and used for decades. The novelty is that instead of analyzing a single CV - as it was done in the previous studies [2-5], we evaluate a set of CVs with different scan rates together. In the present way of analysis, there is an implicit scan rate dependence involved; this is how we can extrapolate to ideally kinetics-controlled and transport controlled situations. Here the scan rate plays the role of tuning the ratio of rates of charge transfer and transport, analogously to the role of rotation frequency of an RDE (as expressed by the Koutecky-Levich equation, see Ref. 1, Ch.14.4.1) or to the role of frequency in impedance measurements (when Warburg term of the Faradaic impedance shrinks to yield the charge transfer resistance at the high frequency limit, see Ref. 1, Ch.10.4.1).

2. Eq.8 is derived for the case when only one form of the redox system is present in the solution bulk. However, it works well also with both forms are present. As it will be shown in a forthcoming publication, both the cathodic and the anodic branch of the $j_{\text {inf }}(E)$ polarization curve appear; and both plateaus of $M_{r e v}(E)$ appear with values expected from the input data and the correspondig theory [11].

3. For an lms fit at least three CVs of different scan rates are needed. However, it is just sufficient to measure two CVs with different scan rates, yielding current densities $j_{1}$ and $j_{2}$, and semiintegrals $M_{1}$ and $M_{2}$. The straight lines are determined by two points at each potentials. Then

$$
j_{\text {inf }}=\frac{M_{2} \cdot j_{1}-M_{1} \cdot j_{2}}{M_{2}-M_{1}} \quad \text { and } \quad M_{\text {rev }}=-\frac{M_{2} \cdot j_{1}-M_{1} \cdot j_{2}}{j_{2}-j_{1}}
$$

(9a and 9b)

4. The $k(E)$ determination of Imbeaux and Savéant (cf. Eq.3) is evidently a much more correct way of determination of parameters of kinetics, $\left(k_{0}, E_{0}\right.$ and $\left.\alpha_{\mathrm{a}}\right)$ and of diffusion $\left(D_{\text {red }}\right)$ than the widely used method of calculating $k_{0}$ from CV peak separation [12] (see also Ch. 6.5.2 of [1]). The superiority can be traced back to the difference that the complete CV is used for the analysis - rather than single (albeit characteristic) data points only. The analysis presented in this paper uses the data of a couple of CVs together, hence probably leads to more accurate values of rate coefficients than all previous methods.

5. Throughout this paper $\mathrm{CV}$ experiments were considered, and the scan-rate independency of $j_{\text {inf }}(E)$ and $M_{r e v}(E)$ was demonstrated. However, the condition of linearity of the $E(t)$ function has not been used up in the derivation, thus Eqs. 7 to 9 apply also for any non-linear $E(t)$ potential programs. Hence, generally speaking, the $j_{\text {inf }}(E)$ and $M_{r e v}(E)$ are the potential-program independent response functions of the system. Analogously, impedance and related functions such as admittance are also potential-program-independent response functions, because they do not „remember" the actual form of the potential perturbation (sinusoidal, white-noise, step function, Dirac-delta, etc) by which they have been determined. Based on the magnitude of $E(t), j_{\text {inf }}(E)$ together with $M_{r e v}(E)$ and impedance are the potential program independent large and small signal response functions, respectively.

If $j_{\text {inf }}(E)$ and $M_{r e v}(E)$ are the potential-program independent functions, then their derivative with respect to potential are also potential program independent ones. $\partial j_{\text {inf }} / \partial E$ is a reciprocal of a charge transfer resistance, provided that diffusional hindrance of charge transfer is negligible. $\partial M_{\text {rev }} / \partial E$ is known to be the coefficient of the Warburg-admittance in a reversible system [13]. This is how the large-signal and small-signal response functions (CV and impedance, respectively of the quasi-reversible systems) are related to each other through their potential-program invariant forms. 


\section{Conclusions}

Results presented here show how to transform quasi-irreversible CVs to yield two scan-rate independent functions. One of them is characteristic to charge transfer kinetics, the other to the diffusional flux. From algebra point of view, Eq. 7 is a simple combination of five well-known equations pertinent to the $\mathrm{CV}$ of quasi-reversible redox systems. The novelty of the present approach is in the use of Eq. 7: As in Eq. 8, we make use the implicit scan rate dependence of the current and of its semiintegral at a constant potential. In other words, the two functions are determined from more-than-one CVs of different scan rates rather than from a single CV.

\section{Acknowledgements:}

The simulated CVs have been generated by a program written by Dr. Soma Vesztergom (Eötvös University, Budapest). The research within project No. VEKOP-2.3.2-16-2017-00013 was supported by the European Union and the State of Hungary, co-financed by the European Regional Development Fund. Additional support of the Hungarian Science Research Fund OTKA (No. K112034) is acknowledged.

\section{References:}

$1 \quad$ A.J. Bard and L.R. Faulkner, Electrochemical Methods, $2^{\text {nd }}$ ed. Wiley, 2001.

2 J.C. Imbeaux and J.M. Savéant, Convolutive potential sweep voltammetry, I. Introduction, $J$. Electroanal. Chem. 44 (1973) 169.

3 K.B. Oldham, J.C. Myland, Extracting parameter values from quasireversible cyclic voltammograms, J Solid State Electrochem. 16 (2012) 3691.

4 K.B. Oldham, J.C. Myland, A.M. Bond, Electrochemical science and technology: fundamentals and applications, Wiley, 2012, ISBN 978-0-470-71085-2, Ch.12.

$5 \quad$ A.Neudeck, F.Marken, R.G. Compton, Complex electron transfer kinetic data from convolution analysis of cyclic voltammograms. Theory and application to diamond electrodes, Electroanalysis 11 (1999) 1149.

6 K.B. Oldham, A signal-independent electroanalytical method, Anal. Chem, 44 (1972) 196.

7 S. Vesztergom, A short introduction to digital simulations in electrochemistry: Simulating the Cottrell experiment in NI LabVIEW, Journal of Electrochemical Science and Engineering, (in press, 2018).

8 S.Vesztergom, N. Barankai, N. Kovács, M. Ujvári, T. Wandlowski, and G.G. Láng, Rotating ring-disk electrode with dual dynamic potential control: theory and practice, Acta Chim. Slov. 61 (2014) 223.

9 S.Vesztergom, N.Barankai, N. Kovács, M.Ujvári, P.Broekmann, H.Siegenthaler, G.G.Láng, Electrical cross-talk in rotating ring-disk experiments, Electrochem. Comm. 68 (2016) 54.

10 T. Pajkossy, L. Nyikos, Fast algorithm for differintegration, J. Electroanal. Chem. 179 (1984) 65.

11 K.B. Oldham, G.D. Zoski, Effect of semioperators on reversible cyclic voltammograms, Anal. Chem. 52 (1980), 2116.

12 R.S. Nicholson, Theory and application of cyclic voltammetry for measurement of electrode reaction kinetics, Anal. Chem. 37 (1965) 1351.

13 T. Pajkossy, Potential program invariant representation of voltammetric measurement results of reversible redox couples, J. Electroanal. Chem. 422 (1997) 13. 